\title{
Topic Modeling in Embedding Spaces for Depression Assessment
}

\author{
Maxime D. Armstrong, Diego Maupomé and Marie-Jean Meurs* \\ Université du Québec à Montréal, Montréal, QC, Canada
}

\begin{abstract}
This paper presents an investigation of topic modeling in embedding spaces performances in the context of depression assessment. Using the textual content of social media users from the eRisk 2018 dataset, a classification task is performed employing features generated from the Embedded Topic Model. To set contrast with traditional topic modeling, a full comparison with the Latent Dirichlet Allocation model is shown. An extensive range of topics and different preprocessing strategies are studied to demonstrate the efficiency of the models. Our results show a noteworthy improvement in the explored task from the application of the novel topic modeling approach.
\end{abstract}

Keywords: Topic modeling · Word embeddings · Depression assessment

\section{Introduction}

Depression is one of the most pervasive mental disorders, with over 264 million people affected worldwide. [1]. Looking more closely at the Canadian situation, $8 \%$ of adults will experience major depression in their lifetime. [2]. In 2018, nearly one out five health care requests was related to mental health, making it the most common reason for the population to seek assistance [3]. Nevertheless, the stigma encircling mental disorders often leads to treatment avoidance and delays to care, resulting in deficient support and absence of proper expert diagnosis [4]. As better treatments result from early intervention [5, 6], our efforts focus on automatic depression assessment using textual content from social media. Systems using such AI-based methods could embodied better tools to evaluate the mental status of a patient, which could be helpful and time-saving for mental health practitioners. Since understanding an individual's mental condition is essential to the selection of a treatment, tools implementing topic modeling methods would be an asset for the practitioners' interpretation of the results.

The analysis of textual content from social media has proven to be a promising avenue for the automatic assessment of mental health issues, demonstrating efficiency for prediction and classification tasks surrounding depression, suicidality and anxiety disorders [7-10]. Topic modeling also displayed considerable potential for depression-related tasks, including classification. Previous work $[11,12]$ emphasizes the usage of Latent Dirichlet Allocation (LDA) [13] to perform topic extraction in this context, allowing to produce discriminant features and interpretable distributions of words from the induced topics. Word embeddings also demonstrated noteworthy performances on sentiment analysis tasks, such as classifying emotions from texts [14] and evaluating the feelings associated to Twitter posts [15]. Since both topic modeling and word embeddings methods reached noticeable performances, systems combining them, such as the Embedded Topic Model (ETM) [16], might improve the results obtained in depression assessment tasks.

Topic modeling in embedding spaces [16] is hence studied in this work, comparing the traditional and the novel topic modeling approaches in the context of depression risk assessment. A classification task is conducted to evaluate the quantitative performances of the models. The ability to extract discriminant topics related to depression is then investigated. The next Section presents the proposed models and the dataset considered for our experiments. Section 3 describes the performed experiments. Section 4 analyzes the obtained results while Section 5 concludes and proposes some directions for future investigations.

${ }^{*}$ meurs.marie-jean@uqam.ca

This article is (C) 2021 by author(s) as listed above. The article is licensed under a Creative Commons Attribution (CC BY 4.0) International license (https://creativecommons.org/licenses/by/4.0/legalcode), except where otherwise indicated with respect to particular material included in the article. The article should be attributed to the author(s) identified above. 
Table 1. Summary statistics of the dataset from the eRisk2018 shared task.

\begin{tabular}{r|r|r} 
& RISK & CONTROL \\
\hline \# users & 214 & 1493 \\
\# writings & 90222 & 986360 \\
writings / user & 411.6 & 660.7 \\
avg. words / writing & 27.5 & 22.8
\end{tabular}

\section{Models and Dataset}

Models. To demonstrate comparison between both topic modeling approaches, the wellknown LDA [13], previously used in [12], is selected as our baseline. LDA uses Dirichlet distributions within a probabilistic generative process to posit $\mathrm{K}$ topics from a corpus, each one being its own distribution over words. Every document within the corpus is assumed to be a mixture of the extracted topics, the proportion of which depends on the words observed in the said document.

The ETM [16] employs a similar generative process, however using word embeddings to do so. Contrary to the traditional modeling approach, the topics evaluated by the ETM are expressed as embeddings, i.e. vectors, belonging to the embedding space, where the vocabulary is represented. Through its generative process, the ETM computes the distribution of topics over words, where the probability of a word pertained to a topic is estimated across the difference between both of their embeddings.

In both cases, the inferred models evaluate the underlying structure within a corpus, allowing to represent each of the documents as a proportion of topics. Thus, some topics could be discriminant features in tasks such as classification, facilitating the interpretation of results, since their distribution of words over the vocabulary is understandable to humans. Here, a document being the collection of a user's writings, as detailed hereafter, these proportions are then exploited to predict the user's depression risk.

Dataset. The dataset considered for our experiments is selected from the eRisk2018 shared task [17], which comprises the written production of English-speaking Reddit users. It is divided into two classes of users: RISK (positive) and CONTROL (negative). The users included in the RISK class have admitted to having been diagnosed with depression; CONTROL users have not. The users' writings, which are posts or comments on the original website, are extracted from various threads of discussion. It is to be noted that only users are labeled, not their individual writings.

The original dataset was split into a training set and a test set, which showed several imbalances in the class distribution. Thus, both subsets have been combined to form a sole dataset, for which the statistics are displayed in Table 1. For the purpose of our task, a custom balanced split is created, dividing the unified dataset into a training set (80\%), a validation set $(10 \%)$ and a test set $(10 \%)$. In doing so, 1365 documents are retained for training, while the validation and test sets kept respectively 171 documents.

\section{Experiments and Results}

Model configurations. The ETM variant using Pre-fitted Word Embeddings (PWE) is selected, as its interpretability and predictive power obtained the best performances in [16]. Since our corpus neighbours $25 \mathrm{M}$ words, from which the rare occurences are removed for training, the Continuous Bag-of-Words (CBOW) algorithm of Word2Vec [18] is chosen to generate the PWE as it tends to smooth better bigger datasets with frequent words [19]. The ETM retains the default parameters put forward in [16], setting the learning rate with the Adam optimizer [20] and selecting a ReLU activation function. In parallel, the LDA is configured to perform 50 passes through the corpus during training, with its log perplexity evaluated every time. 
Preprocessing \& Training. Two preprocessing strategies were applied on the dataset. First is a regular one, i.e. a word undergoes no alteration after tokenization, as adopted in [16]. Following [21], a stemming strategy is also selected, where a word is stemmed after tokenization. Models trained with the latest one are denoted MODEL-stem, MODEL-reg otherwise. It is to be noted that each user of the dataset is viewed as a sole document for our experiments, where a user's writtings are concatenated to create the latter.

To train a PWE, a preprocessed corpus is generated after the application of a strategy, in which the documents are divided in smaller chunks of 200 words for computational efficiency. Two independent PWE are fitted, one per strategy, ensuring the presence of every token in the embedding spaces during the training of the ETM models. For the training of the topic models, the stop words and short words (3 characters or fewer) are removed in every document prior to tokenization. Also, extremes are filtered, removing words appearing in less than 20 documents or more than $50 \%$ of the documents.

Several numbers of topics have demonstrated efficiency for depression assessment tasks, most of the time ranging between 10 and 50 topics [11, 12, 21]. Alongside, the ETM has shown promising results in topic extraction and interpretability using larger numbers, such as 300 topics. Following these ideas, our models are trained on numbers of topics (K) ranging from 10 to 60 in steps of 10, and from 60 to 300 in steps of 30. After completing the model training, each document is used to generate a vector of topics, representing the topic probabilities related to every user. Following [12], a multilayer perceptron classifier formed of 3 hidden layers is trained on these vectors. The size of each hidden layer is set to 300 neurons for every model.

Evaluation \& Metrics. Beyond goodness of fit, an important aspect of topic models is the interpretability of the extracted topics. A few quantitative measures of the semantic soundness of topic models have been proposed. Topics being probability distributions over the entire vocabulary, these measures often focus on the most likely words of each topic. Topic Coherence (TC) [22], also known as UMass Coherence, measures the conditional $\log$ probability of the $M$ most likely words in a topic. In a complementary manner, Topic Diversity (TD) [16] measures the overlap among the $M$ most likely words across topics. Specifically, TD is computed as the proportion of the unique occurences found unique among such words of every topic. In order to balance these two measures, Model Quality (MQ) is defined as the product of TC and TD. Undermentioned TC and TD are denoted with a @ $M$ mention, indicating the $M$ most likely words used to calculate these. Following [17], the standard metrics of Precision $(\mathrm{P})$, Recall $(\mathrm{R})$ and $\mathrm{F}$-measure $\left(\mathrm{F}_{m}\right)(\beta=1)$ are used for classification.

Results. The results obtained show ETM outperforming LDA both in terms of quality and predictive power. Moreover, ETM better sustain both of these aspects over smaller and larger values of K. Figure 1 displays a close view of the results gained from the models trained to extract smaller counts of topics, as well as the longer trend from 30 to 300 topics.

The ETM-stem model holds the best position for all but one of the proposed metrics, reaching a MQ of .355 and a $\mathrm{F}_{m}$ of .611. The leading $\mathrm{P}$ of 0.944 is detained by the LDA-stem model. However, the ETM-stem model is also relatively competent at this level, its highest $\mathrm{P}$ being .889. The foremost performances of this model were achieved with four different $\mathrm{K}$ topics, which are 10, 50, 150 and 270. Table 2 shows a comparison between the models at each of these values of $\mathrm{K}$.

While the split used in our experiments is different from the original eRisk one, the ETM performances are noteworthy compared to the results obtained with LDA in [12]. Table 3 displays the contrast between our LDA-stem and ETM-stem models, holding the best metrics within our experiments, and the previously proposed system. 

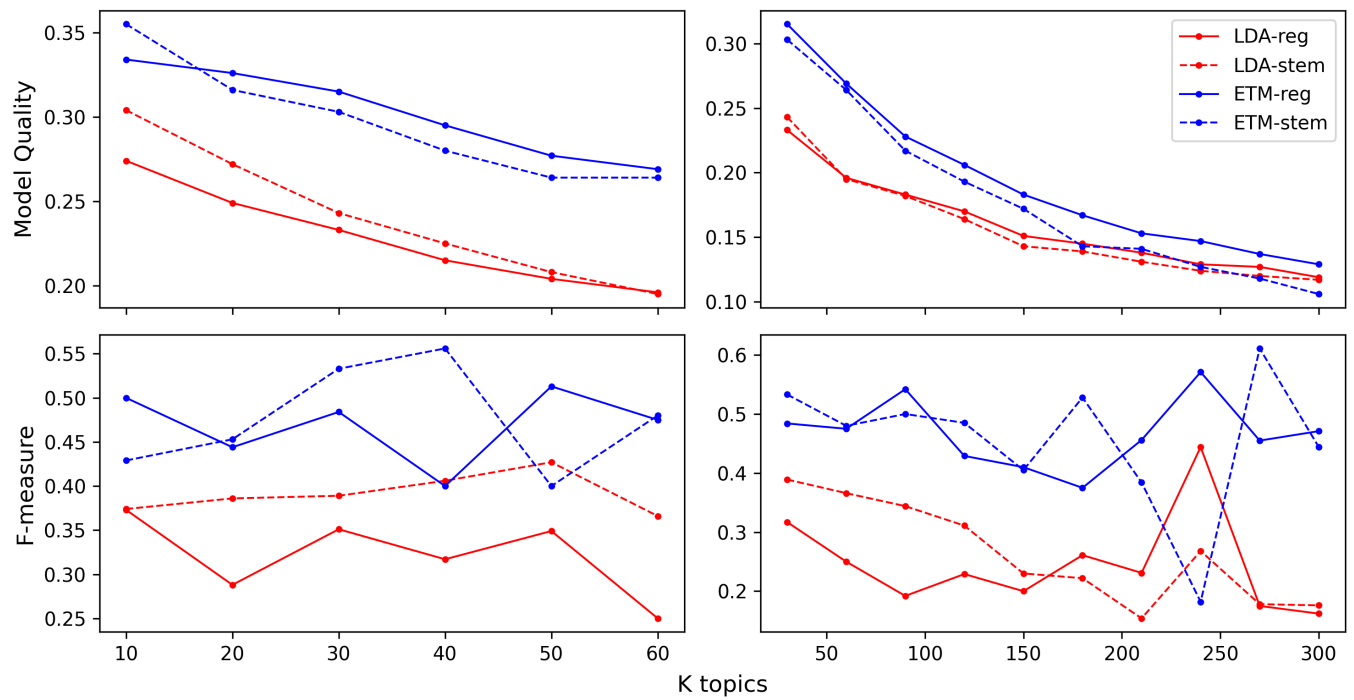

Figure 1. Evolution of Model Quality (TC@10, TD@25) \& F-measure according to K topics.

Table 2. Results for ETM and LDA models for $\mathrm{K}=10,50,150 \& 270$.

\begin{tabular}{c|c|ccc|ccc} 
Model & $\mathrm{K}$ & $\mathrm{TC} @ 10$ & $\mathrm{TD} @ 25$ & $\mathrm{MQ}$ & $\mathrm{P}$ & $\mathrm{R}$ & $\mathrm{F}_{m}$ \\
\hline LDA-reg & 10 & .306 & .896 & .274 & .268 & .611 & .373 \\
& 50 & .273 & .746 & .204 & .221 & .833 & .349 \\
& 150 & .266 & .566 & .151 & .116 & .722 & .200 \\
& 270 & .252 & .505 & .127 & .101 & .667 & .175 \\
\hline LDA-stem & 10 & .334 & .908 & .304 & .233 & $\mathbf{. 9 4 4}$ & .374 \\
& 50 & .291 & .714 & .208 & .281 & .889 & .427 \\
& 150 & .268 & .533 & .143 & .137 & .722 & .230 \\
& 270 & .266 & .450 & .120 & .103 & .667 & .178 \\
\hline ETM-reg & 10 & .343 & $\mathbf{. 9 7 2}$ & .334 & .500 & .500 & .500 \\
& 50 & .291 & .714 & .208 & .281 & .889 & .427 \\
& 150 & .319 & .575 & .183 & .267 & .889 & .410 \\
& 270 & .317 & .434 & .137 & .385 & .556 & .455 \\
\hline ETM-stem & 10 & $\mathbf{. 3 6 5}$ & $\mathbf{. 9 7 2}$ & $\mathbf{. 3 5 5}$ & .600 & .333 & .429 \\
& 50 & .339 & .781 & .264 & $\mathbf{. 7 1 4}$ & .278 & .400 \\
& 150 & .324 & .530 & .172 & .262 & .889 & .405 \\
& 270 & .320 & .369 & .118 & .611 & .611 & $\mathbf{. 6 1 1}$
\end{tabular}

Table 3. Comparative results between the LDA model from Maupomé and Meurs [12], LDA-stem and ETM-stem. For our models, only those with the best $\mathrm{F}_{m}$ are displayed.

\begin{tabular}{c|c|ccc} 
Model & $\mathrm{K}$ & $\mathrm{P}$ & $\mathrm{R}$ & $\mathrm{F}_{m}$ \\
\hline UQAMA [12] & 30 & .32 & .62 & .42 \\
LDA-stem & 50 & .281 & .889 & .427 \\
ETM-stem & 270 & .611 & .611 & .611
\end{tabular}

\section{Discussion}

In view of the results, the models ability to extract topics as astute depression-related features is investigated. Mann-Whitney $U$ test is retained to evaluate the most discriminant topics for each model, for its capacity to assess samples in a binary non-parametric context [23]. To perform the test, the per-topic log probability distributions of the positive and negative users of the training set are selected. Table 4 presents the most discriminant topics gained from the experiment. The tokens displayed suggest that the models are indeed 
Table 4. Top tokens from the most discriminant topics containing the token depression

(-reg) or depress- (-stem) among its 10 first ones. $\mathrm{R}$ stands for the topic rank. K = 270.

\begin{tabular}{c|c|l} 
Model & $\mathrm{R}$ & Tokens \\
\hline LDA-reg & 1 & depression, pain, anxiety, mental, relationship, doctor, health, therapy, haha, depressed \\
LDA-stem & 1 & depress-, emot-, anxieti-, mental-, attract-, medic-, sexual-, disord-, suffer-, neg- \\
ETM-reg & 4 & mental, brain, depression, comfortable, physical, pain, fitness, disabled, dont, injury \\
ETM-stem & 1 & weight-, depress-, reward-, downvot-, crit-, meta-, miser-, quit-, somewhat-, neg-
\end{tabular}
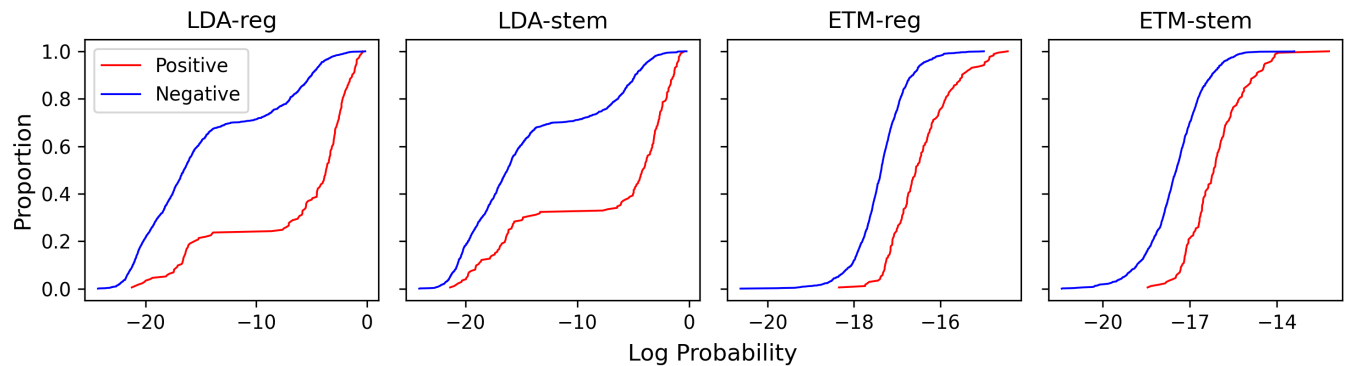

Figure 2. Empirical distribution function of the most discriminating depression-related topic extracted per model. The y-axis shows the proportion of users for which the log probability for the selected topic is less than or equal to the value in $\mathrm{x}$. $\mathrm{K}=270$.

able to extract an underlying structure related to depression, which could lead to the creation of shrewd features for classification. Further examination is carried out by analyzing the aforesaid per-topic log probability distributions for the selected discriminant topics, as shown in Figure 2. The curves observed for the topics extracted by the ETM show a distinct and concise evolution of the distribution, with similarities to a normal one. A closer look of the ETM-stem graph reveals a significant separation around the log probability -17, where about $80 \%$ of the negative users are under or reaching this value, versus $20 \%$ for the positive users. On the opposite, the topics obtained by the LDA models fail to attribute clear and differentiating log probabilities to either of the positive or negative users. The LDA-stem curves plateau around log probabilities -15 to -7 , indicating that the users find themselves largely before or after these points, without a notable division. These findings highlight the ETM aptness to produce astute features, which could explain its predictive power for classification. Furthermore, this suggests that the vocabulary dividing positive users from negative users includes words that are further removed from a lexicon typically associated with depression, as suggested in the Table 4 . Such information could prove to be a relevant addition when interpreting the obtained results, particularly when an individual's mental condition is assessed by a mental health professional.

\section{Conclusion}

The ETM and LDA models were tested on a classification task related to depression assessment. The most performing model, ETM-stem, present significant results both in quality and prediction. The features produced from the topics extracted by the ETM models exhibit a notable dividing power, being able to better differentiate between positive and negative users than the LDA models. Overall, the stemming strategy holds the best position with the ETM-stem and LDA-stem holding the six leading metrics scores.

Future work may include feature selection, based on their precision and recall capabilities. Also, in-depth work on vocabulary analysis to better distinguish between the two types of users could be conducted.

Reproducibility. The source code of the proposed systems is licensed under the GNU GPLv3. The datasets are provided on demand by the eRisk organizers. 


\section{Acknowledgements}

The authors would like to thank Fanny Rancourt for useful discussions and suggestions. This research was enabled in part by support provided by Calcul Québec and Compute Canada. MJM acknowledges the support of the Natural Sciences and Engineering Research Council of Canada [NSERC Grant number 06487-2017] and the Government of Canada's New Frontiers in Research Fund (NFRF), [NFRFE-2018-00484].

\section{References}

[1] S. L. James, D. Abate, K. H. Abate, S. M. Abay, C. Abbafati, N. Abbasi, H. Abbastabar, F. Abd-Allah, J. Abdela, A. Abdelalim, et al. "Global, Regional, and National Incidence, Prevalence, and Years Lived with Disability for 354 Diseases and Injuries for 195 Countries and Territories, 1990-2017: A Systematic Analysis for the Global Burden of Disease Study 2017". In: The Lancet (2018).

[2] Canadian Mental Health Association. Fast Facts about Mental Illness. 2020.

[3] Statistics Canada. Care Counts: Receiving Care for a Mental Illness, 2018. 2020.

[4] C. Henderson, S. Evans-Lacko, and G. Thornicroft. "Mental Illness Stigma, Help Seeking, and Public Health Programs". In: American journal of public health (2013).

[5] C. Arango, C. M. Díaz-Caneja, P. D. McGorry, J. Rapoport, I. E. Sommer, J. A. Vorstman, D. McDaid, O. Marín, E. Serrano-Drozdowskyj, R. Freedman, et al. "Preventive Strategies for Mental Health". In: The Lancet Psychiatry (2018).

[6] Canadian Mental Health Association. Early Intervention. 2020.

[7] J. Ive, G. Gkotsis, R. Dutta, R. Stewart, and S. Velupillai. "Hierarchical Neural Model with Attention Mechanisms for the Classification of Social Media Text Related to Mental Health". In: Workshop on Computational Linguistics and Clinical Psychology. 2018.

[8] R. M. Merchant, D. A. Asch, P. Crutchley, L. H. Ungar, S. C. Guntuku, J. C. Eichstaedt, S. Hill, K. Padrez, R. J. Smith, and H. A. Schwartz. "Evaluating the Predictability of Medical Conditions from Social Media Posts". In: PLOS ONE ().

[9] G. Coppersmith, M. Dredze, C. Harman, K. Hollingshead, and M. Mitchell. "CLPsych 2015 Shared Task: Depression and PTSD on Twitter". In: Workshop on Computational Linguistics and Clinical Psychology. 2015.

[10] J. H. Shen and F. Rudzicz. "Detecting Anxiety through Reddit". In: Workshop on Computational Linguistics and Clinical Psychology. 2017.

[11] P. Resnik, W. Armstrong, L. Claudino, T. Nguyen, V.-A. Nguyen, and J. Boyd-Graber. "Beyond LDA: Exploring Supervised Topic Modeling for Depression-Related Language in Twitter". In: Workshop on Computational Linguistics and Clinical Psychology. 2015.

[12] D. Maupomé and M.-J. Meurs. "Using Topic Extraction on Social Media Content for the Early Detection of Depression." In: CLEF (Working Notes) 2125 (2018).

[13] D. M. Blei, A. Y. Ng, and M. I. Jordan. "Latent Dirichlet Allocation". In: Journal of machine Learning research (2003).

[14] M. Giatsoglou, M. G. Vozalis, K. Diamantaras, A. Vakali, G. Sarigiannidis, and K. C. Chatzisavvas. "Sentiment Analysis Leveraging Emotions and Word Embeddings". In: Expert Systems with Applications (2017).

[15] D. Tang, F. Wei, N. Yang, M. Zhou, T. Liu, and B. Qin. "Learning Sentiment-Specific Word Embedding for Twitter Sentiment Classification". In: Annual Meeting of the Association for Computational Linguistics. 2014.

[16] A. B. Dieng, F. J. Ruiz, and D. M. Blei. "Topic Modeling in Embedding Spaces". In: Transactions of the Association for Computational Linguistics (2020).

[17] D. E. Losada, F. Crestani, and J. Parapar. "Overview of eRisk 2018: Early Risk Prediction on the Internet". In: CLEF (Working Notes). 2018.

[18] T. Mikolov, I. Sutskever, K. Chen, G. Corrado, and J. Dean. "Distributed Representations of Words and Phrases and their Compositionality". In: arXiv preprint arXiv:1310.4546 (2013).

[19] T. Mikolov, K. Chen, G. Corrado, and J. Dean. "Efficient Estimation of Word Representations in Vector Space". In: arXiv preprint arXiv:1301.3781 (2013).

[20] D. P. Kingma and J. Ba. "Adam: A Method for Stochastic Optimization". In: arXiv preprint arXiv:1412.6980 (2014).

[21] D. Maupomé, M. D. Armstrong, R. Belbahar, J. Alezot, R. Balassiano, M. Queudot, S. Mosser, and M.-J. Meurs. "Early Mental Health Risk Assessment through Writing Styles, Topics and Neural Models". In: CLEF (Working Notes). 2020. 
[22] D. Mimno, H. Wallach, E. Talley, M. Leenders, and A. McCallum. "Optimizing Semantic Coherence in Topic Models". In: International Conference on Empirical Methods in Natural Language Processing. 2011.

[23] N. Nachar et al. "The Mann-Whitney U: A Test for Assessing whether Two Independent Samples Come from the Same Distribution". In: Tutorials in quantitative Methods for Psychology (2008). 